\title{
Evaluation of Applying Surface Simplification Techniques in Medical Volume Data
}

\author{
Zainab Al-Rahamneh \\ Department of Computer Information Systems \\ Al-Balqa Applied University \\ Salt, Jordan
}

\author{
Asma'a Khtoom \\ Department of Computer Information Systems \\ Al-Balqa Applied University \\ Salt, Jordan
}

\author{
Mohammad Ryalat \\ Department of Computer Science \\ Al-Balqa Applied University \\ Salt, Jordan
}

\begin{abstract}
Medical volume data such as MRI and CT images consist of a large number of voxels. Thus, the process of displaying, storing and transmission of medical volume data is a big challenge in the biomedical field. Applying surface simplification techniques to reduce the size occupied by medical images is considered as one of the most common approachs to overcome this challenge. However, not all of the surface simplification techniques are accurate enough to be used in the medical fields. This paper aims to evaluate the impact and the accuracy of applying the Uniform Mesh Resampling (UMR) technique and the Quadric Edge Collapse Decimation (QECD) technique. Moreover, this study investigates Poisson Surface Reconstruction (PSR) technique and sets experimentally the optimal offsetting value of this technique. Two real medical benchmark datasets are used in this study to evaluate the experimental work. The outcomes indicate clearly that the use of QECD as a surface simplification technique achieves competitive results when used with medical volume data.
\end{abstract}

\section{General Terms}

Biomedical Engineering, Computer Algorithms for Medical Images

\section{Keywords}

Medical Volume Data, Medical Images, Surface Simplification, Dice Coefficient, Stl Files

\section{INTRODUCTION}

\subsection{Medical Volume Data}

Volume data in medicine and medical volume images, such as Computed Tomography (CT) and Magnetic Resonance Imaging (MRI), play a key role in monitoring the progression of different diseases [14, 10]. These type of medical images help the physician to track the efficiency of medication and adapt protocols as needed [8].
Table 1. Average file sizes of various imaging modalities.

\begin{tabular}{|l|l|r|}
\hline Image type & width x height & File size \\
\hline Digital radiography & $3000 \times 3000$ & $\sim 18 \mathrm{MB}$ \\
Digital mammography & $3328 \times 4096$ & $\sim 27 \mathrm{MB}$ \\
Computed radiography & $3520 \times 4280$ & $\sim 30 \mathrm{MB}$ \\
\hline
\end{tabular}

Medical volume data are ordered as a Cartesian grid named voxels. Since different imaging modalities need a large number of voxels to be electronically stored, this leads to make the following operations time-consuming and requires the need for high computational resources:

* Medical image registration

* Medical image segmentation

* Exploring and diagnosis of volume data on computers

* Transferring of medical volume images

Table 1], which is adapted from [3], tabulates the average size of files for images generated by different imaging modalities. It is noteworthy that the values presented in the third column of Table 1 are per image. Since there are dozens and even hundreds of images for each patient, then one stack of these medical images for one patient needs several gigabytes to be stored and processed.

The process of displaying and transferring of medical volume data (i.e., medical volume images) are considered a big challenge in the biomedical field [5]. One common solution of this challenge is the reducing of the file size of medical volume data. Reducing the size, which is normally performed by surface simplification, aims to generate, in smaller size, an approximation of the original volume image. The process of reduction the size using surface simplification techniques over medical data has the following attributes:

* It has a sufficiently significant effect on the speed of the processing particularly on low-end servers [7]

* It is a fundamental approach when computing resources (such as RAM, CPU and graphics card) are limited. 


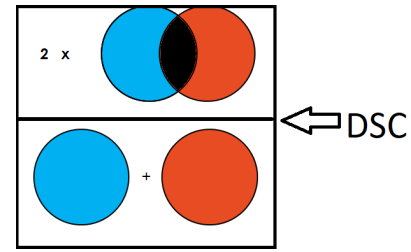

Fig. 1. Graphical illustration of Dice similarity coefficient.

Table 2. Description of datasets evaluated in our study.

\begin{tabular}{cllll}
\hline Form & provider & Attributes & \\
\hline \hline Pelvis & & & & \\
\hline STL file & $\begin{array}{l}\text { Able Software Corpo- } \\
\text { ration (USA) }\end{array}$ & $\begin{array}{l}\text { Mesh } \\
\text { (Pelvis) } \\
\text { vertices) }\end{array}$ & surface/ & FileSize \\
Knee & & & & \\
\hline STL file & $\begin{array}{l}\text { The Biomedical 3-D } \\
\text { Printing Community } \\
\text { (embodi3D LLC) }\end{array}$ & Mesh surface/ Knee File-size \\
& $=2665 \mathrm{~KB}$ (26651 vertices)
\end{tabular}

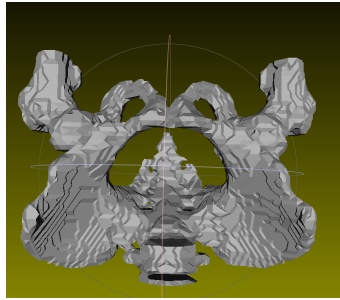

(a)

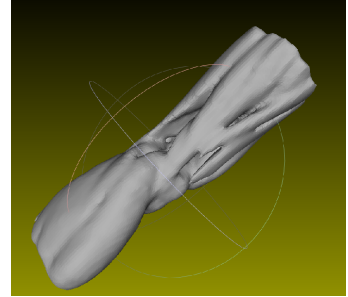

(b)
Fig. 2. The datasets used in this study. (a): surface mesh represents real pelvis. (b): surface mesh represents real knee.

* It is employed to export three-dimensional medical data to manufacture physical biomodels [7].

\subsection{Dice Similarity Coefficient}

Dice Similarity Coefficient (DSC), which was presented in [4], is one of the most common similarity metrics that are utilized to evaluate between two images. It is basically calculated, as illustrated in Figure 1. by multiplying two by the area of overlap (i.e., intersection area) divided by the whole number of pixels (i.e., union area). DSC is one of the widely-accepted metrics used in the medical imaging community [12 13] and therefore it will be used in this study for evaluation of the experiments.

The remaining of this paper is organized as follows: Section 2 describes the materials and datasets used in this study. Section 3 presents the experimental works, where Section 3.1 clarifies the effect of applying surface simplification techniques to reduce the size of medical images, and Section 3.2 presents the optimal setting of some parameters. Section 4 concludes this study and highlights the main contributions.

\section{MATERIALS AND DATASETS}

This section describes the two datasets that are employed in this study to demonstrate and evaluate the experimental work. The details, attributes and source of these datasets are illustrated in Table 2

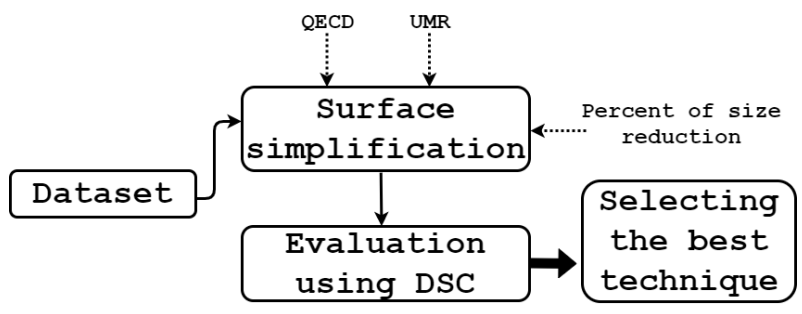

Fig. 3. General Schema of the Evaluation Process.

The two datasets, the pelvis and the knee, are illustrated in Figure 2 (a) and 2 b), respectively. The pelvis dataset was imported from [1], where the knee dataset was imported from [2]. The two datasets are generated from stacks of Computed Tomography images and prepared as STereoLithography files (i.e. stl files).

\section{EXPERIMENTAL WORKS}

Numerous techniques are employed in research to reduce the size of medical volume images. These techniques are also named downsampling techniques. The general schema of the experimental work is illustrated in Figure 3 The experimental work in this study involves two separated parts:

(1) In the first part, a comparison between the Uniform Mesh Resampling (UMR) technique and the Quadric Edge Collapse Decimation (QECD) technique [6] [9] is performed. The comparison in this part aims to find which one of the two techniques achieves a higher degree of similarity. This involves the use of various reduced versions of medical volume images. Ten reduced forms of various sizes were produced using UMR approach and other ten reduced forms were produced using QECD approach. This has been applied for each dataset. The DSC was calculated over each corresponding versions (i.e., those that have the same percentage of reduction) to specify the technique that reaches to a higher degree of similarity. Regarding UMR approach, a new mesh is created which represents a resampled mesh of the original mesh. In this context, the proportion of resampling correlates with the value of precision.

(2) In the second part, the aim of the experimental work is to find the best offsetting-value, which is an essential parameter for Poisson Surface Reconstruction (PSR) technique [11]. Nine different values of offsetting value were evaluated for each dataset for the sake of conducting a comparison between the generated surfaces. This in turn will lead to find the optimal offsetting value.

\subsection{Part (I): Comparing QECD and UMR as Surface-Simplification Techniques}

DSC is employed in this part to evaluate the accuracy of the QECD technique against the UMR technique. Table 3 presents the similarity degree between the original object and its reduced forms. The outcomes presented in Table 3 represent the implementation of the QECD technique over the Pelvis and the Knee datasets. The experiments are performed over 11 different reduced forms of the same organ while each row represents one case. To clarify, the second row, for example, displays the DSC between the original object and a reduced version of this object which has a size equals $90 \%$ of the original one.

The table tabulates, for each one of the 11 cases, the Stl file size in kilobyte, the number of vertices of the mesh, the number of faces, 
Table 3. Evaluating DSC on different size-reduced versions (surface-simplified). (using QECD approach)

\begin{tabular}{llllll}
\hline Case\# & Percent & F-Size & Vertices & faces & DSC \\
\hline Pelvis & & & & & \\
1 & Same & 750 & 7,630 & 15,400 & $\mathbf{1 . 0 0 0 0}$ \\
2 & $90 \%$ & 675 & 6,860 & 13,820 & $\mathbf{0 . 9 9 7 0}$ \\
3 & $80 \%$ & 600 & 6,100 & 12,281 & $\mathbf{0 . 9 9 1 0}$ \\
4 & $70 \%$ & 525 & 5,330 & 10,800 & $\mathbf{0 . 9 7 7 1}$ \\
5 & $60 \%$ & 450 & 4,560 & 9,220 & $\mathbf{0 . 9 6 2 0}$ \\
6 & $50 \%$ & 375 & 3,800 & 7,680 & $\mathbf{0 . 9 3 9 0}$ \\
7 & $40 \%$ & 300 & 3,041 & 6,151 & $\mathbf{0 . 9 2 4 0}$ \\
8 & $30 \%$ & 225 & 2,270 & 4,606 & $\mathbf{0 . 9 1 5 0}$ \\
9 & $20 \%$ & 150 & 1,510 & 3,081 & $\mathbf{0 . 9 0 2 0}$ \\
10 & $10 \%$ & 75 & 750 & 1,540 & $\mathbf{0 . 8 7 4 0}$ \\
11 & $1 \%$ & 8 & 74 & 160 & $\mathbf{0 . 6 0 9 7}$ \\
& & & & & \\
Knee & & & & & \\
12 & Same & 2,670 & 26,660 & 53,320 & $\mathbf{1 . 0 0 0 0}$ \\
13 & $90 \%$ & 2,350 & 23,990 & 47,990 & $\mathbf{0 . 9 9 8 7}$ \\
14 & $80 \%$ & 2,140 & 21,325 & 42,661 & $\mathbf{0 . 9 9 7 0}$ \\
15 & $70 \%$ & 1,830 & 18,660 & 37,325 & $\mathbf{0 . 9 9 5 0}$ \\
16 & $60 \%$ & 1,570 & 15,990 & 31,990 & $\mathbf{0 . 9 9 3 0}$ \\
17 & $50 \%$ & 1,340 & 13,331 & 26,662 & $\mathbf{0 . 9 9 1 1}$ \\
18 & $40 \%$ & 1,051 & 10,664 & 21,331 & $\mathbf{0 . 9 8 9 1}$ \\
19 & $30 \%$ & 787 & 7,996 & 15,999 & $\mathbf{0 . 9 8 7 2}$ \\
20 & $20 \%$ & 527 & 5,331 & 10,671 & $\mathbf{0 . 9 8 5 2}$ \\
21 & $10 \%$ & 263 & 2,662 & 5,337 & $\mathbf{0 . 9 8 2 1}$ \\
22 & $1 \%$ & 27 & 263 & 537 & $\mathbf{0 . 9 5 2 1}$ \\
\hline & & & & &
\end{tabular}

and the value of DSC in either the case of filling the slices or with no fill.

It is obvious from Table 3 that there is a positive correlation between the file size and the value of DSC. This is reasonable since reducing the size by performing surface-simplification will lead to lose some details in the generated form, and consequently will decrease the value of the DSC metric.

However, it can be observed from Table 3 that even when the size is reduced to be $20 \%$ of the original size, the DSC gives $90.13 \%$ and $98.41 \%$ for the pelvis and Knee datasets, respectively.

Table 4 tabulates the DSC between the original object and its reduced forms when it was evaluated over the Pelvis and the Knee datasets. The outcomes presented in Table 4 are related to the UMR technique. The experiments are repeated again over 15 resized approximations. UMR, which is used as a surface simplification technique, has a required parameter, named precision, that should be set. Each row in Table 4 represents a different value of precision. The term precision represents the size of cell. Larger cells generates worse precision and vice versa. It is obvious from Table 4 that there is a negative correlation between precision and the value of DSC. While the value of precision increases, the value of DSC decreases. This behavior is completely expected since generating smaller cell produces better value of precision.

Figure 4 displays, using bar charts, a comparison between UMR and QECD in terms of quality of the surface simplification. As illustrated in Figure 4 QECD approach achieves a higher degree of similarity (i.e., DSC) than the UMR approach for the same percentage of reduction (i.e., the same file size). This enforces the fact which was presented in [6]. This conclusion involves all the different sizes of files (i.e., all test cases) which indicate clearly that QECD technique is more convenient than UMR to used in medical fields to reduce the size of the mesh and to approximate volume of medical images. In addition to what mentioned before, the stability of results generated
Table 4. Evaluating DSC on different size-reduced versions (surface-simplified). (using UMR approach)

\begin{tabular}{llllll}
\hline Case\# & Precision & F-Size & Vertices & faces & DSC \\
\hline Pelvis & & & & & \\
1 & Same & 750 & 7,630 & 15,400 & $\mathbf{1 . 0 0 0 0}$ \\
2 & 1.5 & 747 & 7617 & 15280 & $\mathbf{0 . 8 8 5 8}$ \\
3 & 2.0 & 403 & 4124 & 8247 & $\mathbf{0 . 8 5 5 8}$ \\
4 & 2.5 & 241 & 2441 & 4928 & $\mathbf{0 . 8 2 4 7}$ \\
5 & 3.0 & 165 & 1668 & 3358 & $\mathbf{0 . 7 8 1 7}$ \\
6 & 3.5 & 111 & 1135 & 2270 & $\mathbf{0 . 7 4 6 1}$ \\
7 & 4.0 & 81 & 824 & 1652 & $\mathbf{0 . 7 1 0 8}$ \\
8 & 4.5 & 63 & 654 & 1284 & $\mathbf{0 . 6 5 6 8}$ \\
9 & 5.0 & 46 & 487 & 934 & $\mathbf{0 . 6 1 8 1}$ \\
10 & 5.5 & 34 & 367 & 694 & $\mathbf{0 . 5 3 5 7}$ \\
11 & 6.0 & 28 & 294 & 560 & $\mathbf{0 . 5 3 4 8}$ \\
12 & 6.5 & 24 & 248 & 472 & $\mathbf{0 . 4 5 0 7}$ \\
13 & 7.0 & 18 & 197 & 362 & $\mathbf{0 . 4 4 1 4}$ \\
14 & 7.5 & 18 & 191 & 366 & $\mathbf{0 . 4 7 1 2}$ \\
15 & 10 & 7 & 82 & 140 & $\mathbf{0 . 2 2 1 1}$ \\
& & & & & \\
Knee & & & & & \\
16 & Same & 2,670 & 26,660 & 53,320 & $\mathbf{1 . 0 0 0 0}$ \\
17 & 1.5 & 605 & 6182 & 12384 & $\mathbf{0 . 9 7 6 5}$ \\
18 & 2.0 & 324 & 3308 & 6624 & $\mathbf{0 . 9 6 7 0}$ \\
19 & 2.5 & 207 & 2112 & 4228 & $\mathbf{0 . 9 5 7 4}$ \\
20 & 3.0 & 139 & 1407 & 2830 & $\mathbf{0 . 9 3 9 1}$ \\
21 & 3.5 & 100 & 1015 & 2034 & $\mathbf{0 . 9 2 3 0}$ \\
22 & 4.0 & 75 & 757 & 1522 & $\mathbf{0 . 9 1 1 0}$ \\
23 & 4.5 & 61 & 623 & 1238 & $\mathbf{0 . 8 9 5 2}$ \\
24 & 5.0 & 43 & 438 & 876 & $\mathbf{0 . 8 6 3 7}$ \\
25 & 5.5 & 37 & 378 & 756 & $\mathbf{0 . 8 5 7 8}$ \\
26 & 6.0 & 33 & 332 & 668 & $\mathbf{0 . 8 5 0 4}$ \\
27 & 6.5 & 23 & 234 & 464 & $\mathbf{0 . 8 2 0 6}$ \\
\hline & & & & &
\end{tabular}

by the QECD algorithm is obvious. It is clear from Figure 4 that the relation between the DSC and the file size is more linear for QECD when it is compared with the UMR technique. This confirms again the advantages that can be gained when using the QECD in medical fields in order to reduce the size of meshes.

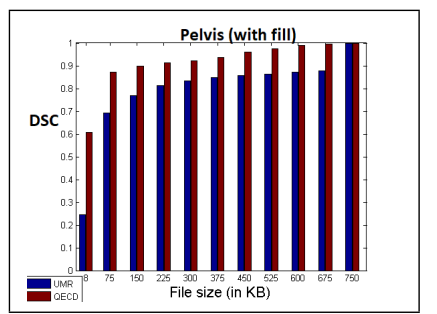

(a)Pelvis with-fill

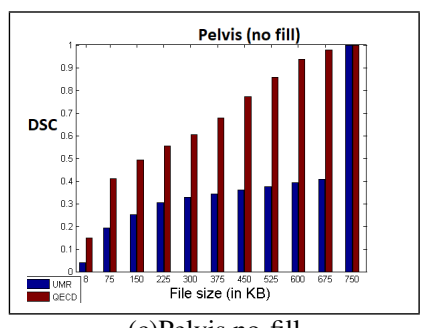

(c)Pelvis no-fill

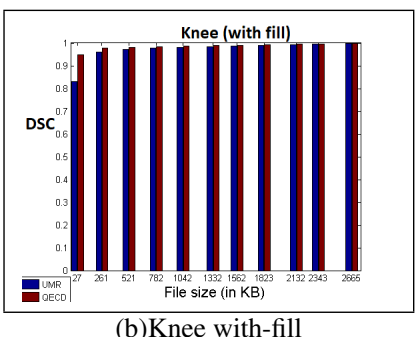

(b)Knee with-fill

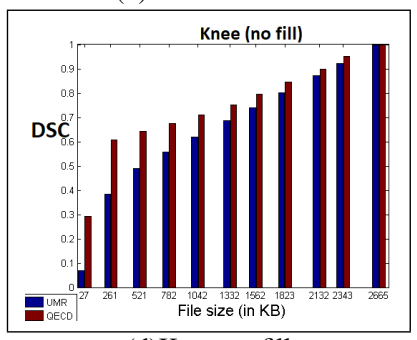

(d)Knee no-fill
Fig. 4. QECD vs. UMR. 


\subsection{Part (II): Specifying experimentally the Best Offsetting Value in Poisson Surface Reconstruction Approach}

Poisson Surface Reconstruction Technique (PSR) has an essential parameter named Correction-value or Offsetting-value $\alpha$. Determining the optimal value of this parameter is time-consuming for many researchers and prone to uncertainty. The aim of the experimental work in this part is to find experimentally the optimal surface offsetting value $\alpha$ of the iso-surface threshold of the PSR technique. The impact of setting different values of $\alpha$ is evaluated and the effect of that on the value of DSC. Table 5 tabulates the value of DSC using different values of the $\alpha$.

Table 5. The DSC of various values of $\alpha$.

\begin{tabular}{llllll}
\hline Case\# & $(\alpha)$ & FSize & Vertices & faces & DSC \\
\hline Pelvis & & & & & \\
1 & Same & 750 & 7,630 & 15,400 & $\mathbf{1 . 0 0 0 0}$ \\
2 & 0.25 & 851 & 8602 & 17201 & $\mathbf{0 . 5 7 4 2}$ \\
3 & 0.50 & 792 & 7995 & 15991 & $\mathbf{0 . 7 4 3 3}$ \\
4 & 0.75 & 721 & 7289 & 14579 & $\mathbf{0 . 8 3 3 1}$ \\
5 & 0.875 & 691 & 6957 & 13917 & $\mathbf{0 . 8 4 1 1}$ \\
6 & 1.0 & 640 & 6530 & 13060 & $\mathbf{0 . 8 4 1 3}$ \\
7 & 1.25 & 567 & 5760 & 11501 & $\mathbf{0 . 7 5 1 2}$ \\
8 & 1.5 & 461 & 4710 & 9391 & $\mathbf{0 . 6 0 3 2}$ \\
9 & 1.75 & 331 & 3373 & 6661 & $\mathbf{0 . 4 1 5 0}$ \\
10 & 2.0 & 197 & 1998 & 3971 & $\mathbf{0 . 2 3 0 2}$ \\
& & & & & \\
Knee & & & & & \\
12 & Same & 2,670 & 26,660 & 53,320 & $\mathbf{1 . 0 0 0 0}$ \\
13 & 0.25 & 456 & 4664 & 9324 & $\mathbf{0 . 7 4 7 8}$ \\
14 & 0.50 & 442 & 4518 & 9032 & $\mathbf{0 . 8 3 1 8}$ \\
15 & 0.75 & 429 & 4392 & 8784 & $\mathbf{0 . 9 0 6 9}$ \\
16 & 0.875 & 427 & 4362 & 8724 & $\mathbf{0 . 9 3 2 4}$ \\
17 & 1.00 & 426 & 4356 & 8712 & $\mathbf{0 . 9 4 2 4}$ \\
18 & 1.25 & 415 & 4240 & 8480 & $\mathbf{0 . 8 8 9 2}$ \\
19 & 1.50 & 386 & 3946 & 7888 & $\mathbf{0 . 7 9 4 3}$ \\
20 & 1.75 & 341 & 3485 & 6970 & $\mathbf{0 . 6 5 7 0}$ \\
21 & 2.00 & 235 & 2401 & 4794 & $\mathbf{0 . 4 5 1 6}$ \\
\hline & & & & & \\
& & & & &
\end{tabular}

Figure 5 plots, for the PSR technique, the value of DSC for diverse values of $\alpha$. The value at which parameter $\alpha$ amounts to the largest DSC possible value are illustrated using red ellipses in Figure 5 It is clear from Figure 5 that selecting a value for $\alpha$ in the interval [00.90-01.00] is the optimal selection to keep the quality of the reduced mesh close to the quality of the original one.

\section{CONCLUSION}

This paper discussed and evaluated the effect of applying surface simplification techniques over medical volume images. The paper presented two contributions. The first one, it compares between two of the common surface simplification techniques and evaluates the quality of the generated reduced versions. The evaluation is performed using the DSC as it is considered one of the widelyaccepted metrics used for medical images. The results indicated clearly that the Quadric Edge Collapse Decimation (QECD) technique exceeds the Uniform Mesh Resampling (UMR) technique in terms of accuracy. The second contribution that this study presented is the setting of the $\alpha$ parameter. The Offsetting-value $\alpha$ is an essential parameter that should be correctly tuned as a preprocessing step for the Poisson Surface Reconstruction Technique (PSR).

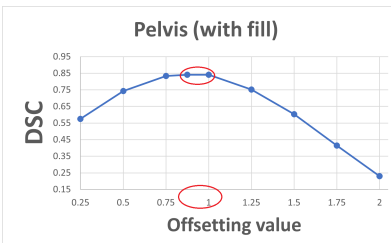

(a) Pelvis (fill)

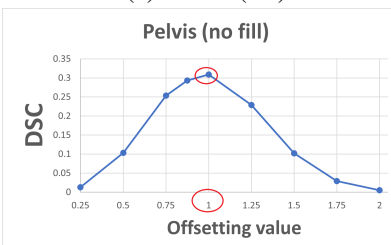

(c) Pelvis (no fill)

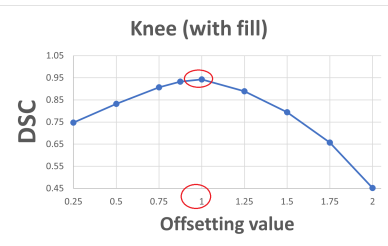

(b) Knee (fill)

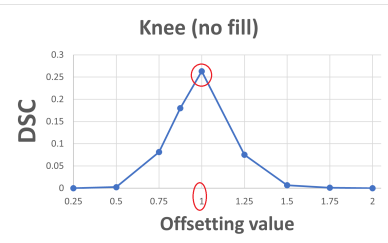

(d) Knee (no fill)
Fig. 5. DSC for various Offsetting values. Red ellipses represent the value at which $\alpha$ amount to the largest value of DSC.

The outcomes of this study confirm the feasibility of using surface simplification techniques, particularly the QECD technique, to store, display, and transmit medical volume data.

\section{REFERENCES}

[1] ABLE SOFTWARE CORP. http://www.ablesw.com/ 3d-doctor/format.html Accessed: 19-01-2017.

[2] The Biomedical 3D Printing Community (embodi3D LLC). https://www.embodi3d.com/. Accessed: 19-01-2017.

[3] Ravi Varma Dandu. Storage media for computers in radiology. The Indian journal of radiology \& imaging, 18(4):287, 2008.

[4] Lee R Dice. Measures of the amount of ecologic association between species. Ecology, 26(3):297-302, 1945.

[5] Feng Ding, Hao Li, Yuan Cheng, and Wee Kheng Leow. Medical volume image summarization. In Applications of Computer Vision (WACV), 2009 Workshop on, pages 1-6. IEEE, 2009.

[6] Michael Garland and Paul S Heckbert. Surface simplification using quadric error metrics. In Proceedings of the 24th annual conference on Computer graphics and interactive techniques, pages 209-216. ACM Press/Addison-Wesley Publishing Co., 1997.

[7] Ian Gibson. Advanced manufacturing technology for medical applications: reverse engineering, software conversion and rapid prototyping. John Wiley \& Sons, 2006.

[8] Daichi Hayashi. Deep learning for lumbar spine mri reporting: A welcome tool for radiologists, 2021.

[9] Paul S Heckbert and Michael Garland. Optimal triangulation and quadric-based surface simplification. Computational Geometry, 14(1):49-65, 1999.

[10] Anastasia Katsavochristou and Dimitrios Koumoulis. Magnetic resonance and ct imaging biomarkers for prediction of acute and chronic radiation-induced xerostomia. Magnetochemistry, 7(1):5, 2021.

[11] Michael Kazhdan, Matthew Bolitho, and Hugues Hoppe. Poisson surface reconstruction. In Proceedings of the fourth Eurographics symposium on Geometry processing, volume 7, 2006. 
[12] Roaa G Mohamed, Noha A Seada, Salma Hamdy, and Mostafa GM Mostafa. An adaptive method for fully automatic liver segmentation in medical mri-images. International Journal of Computer Applications, 179(4):12-18, 2017.

[13] M Seetharama Prasad, T Divakar, B Srinivasa Rao, and $\mathrm{N}$ Raju. Unsupervised image thresholding using fuzzy measures. International Journal of Computer Applications, 27(2):32-41, 2011.

[14] Judith E Spiro, Miriam Rinneburger, Dennis M Hedderich, Mladen Jokic, Hans Christian Reinhardt, David Maintz, Moritz Palmowski, and Thorsten Persigehl. Monitoring treatment effects in lung cancer-bearing mice: clinical ct and clinical mri compared to micro-ct. European radiology experimental, 4(1):1-8, 2020. 